\title{
Lower bounds on Ricci flow invariant curvatures and geometric applications.
}

\author{
Thomas Richard
}

September 25, 2018

\begin{abstract}
We consider Ricci flow invariant cones $\mathcal{C}$ in the space of curvature operators lying between nonnegative Ricci curvature and nonnegative curvature operator. Assuming some mild control on the scalar curvature of the Ricci flow, we show that if a solution to Ricci flow has its curvature operator which satsisfies $\mathrm{R}+\varepsilon \mathrm{I} \in \mathcal{C}$ at the initial time, then it satisfies $\mathrm{R}+K \varepsilon \mathrm{I} \in \mathcal{C}$ on some time interval depending only on the scalar curvature control.

This allows us to link Gromov-Hausdorff convergence and Ricci flow convergence when the limit is smooth and $R+I \in \mathcal{C}$ along the sequence of initial conditions. Another application is a stability result for manifolds whose curvature operator is almost in $\mathcal{C}$.

Finally, we study the case where $\mathcal{C}$ is contained in the cone of operators whose sectional curvature is nonnegative. This allow us to weaken the assumptions of the previously mentioned applications. In particular, we construct a Ricci flow for a class of (not too) singular Alexandrov spaces.
\end{abstract}

\section{Introduction and statement of the results}

In the study of the Ricci flow, various nonnegative curvature conditions have been shown to be preserved, and the discovery of new invariant conditions has often given rise to new geometric applications. One of the most famous occurence of this fact is the discovery by Brendle and Schoen in 7 and independently by Nguyen in 23] of the preservation of nonnegative isotropic curvature, which plays a crucial role in the proof by Brendle and Schoen of the differentiable sphere theorem in 7 .

Once one has understood the behaviour of the Ricci flow assuming the nonnegativity of a certain curvature, it is natural to ask if something can be done under arbitrary lower bounds on this given curvature. Such a work has been done for Ricci curvature in dimension 3 by Simon in [26] and [25]. An important feature of this work is that, in order to control lower bounds on the Ricci curvature along the flow, one has to impose further geometric conditions on the initial manifold. In Simon's work, a non-collapsing assumption is required. Our estimate will rely on an a priori bound on the scalar curvature. 
In order to state the results of this paper, we need some terminology.

Definition 1.1. A nonnegativity condition on the curvature is given by a closed convex cone $\mathcal{C}$ in the space of algebraic curvature operators $S_{B}^{2} \Lambda^{2} \mathbb{R}^{n}$ such that :

- The identity operator $\mathrm{I}: \Lambda^{2} \mathbb{R}^{n} \rightarrow \Lambda^{2} \mathbb{R}^{n}$ lies in the interior of $\mathcal{C}$.

- $\mathcal{C}$ is invariant under the action of $O(n, \mathbb{R})$ on $S_{B}^{2} \Lambda^{2} \mathbb{R}^{n}$ given by :

$$
\langle g . \mathrm{R}(x \wedge y), z \wedge t\rangle=\langle\mathrm{R}(g x \wedge g y), g z \wedge g t\rangle .
$$

Recalls and references on algebraic curvtaure operators are included in Section 2 ,

Given a nonnegativity condition $\mathcal{C}$ and a Riemannian manifold $(M, g)$, we can canonically embed $\mathcal{C}$ in $S_{B}^{2} \Lambda^{2} T_{m} M$ for each $m \in M$, thanks to the $O(n, \mathbb{R})$ invariance of $\mathcal{C}$. We say that $(M, g)$ has $\mathcal{C}$-nonnegative curvature (or $\mathrm{R} \geq_{\mathcal{C}}$ 0 ) if, for each $x \in M$ the curvature operator of $(M, g)$ at $x$ belongs to $\mathcal{C}$. Classical condtions of nonnegative curvature operator, nonnegative sectional curvature, nonnegative Ricci curvature or nonnegative scalar curvature fit in this framework.

Similarly we say that $(M, g)$ has $\mathcal{C}$-curvature bounded from below by $-k \mathrm{I}$ (or $\mathrm{R} \geq_{\mathcal{C}}-k \mathrm{I}$ ) for some $k \in \mathbb{R}$ if for each $x \in M$ the curvature operator $\mathrm{R}$ at $x$ is such that $\mathrm{R}+k \mathrm{I} \in \mathcal{C}$.

We now define a class of nonnegativity condition which behaves well with Ricci flow.

Definition 1.2. A nonnegativity condition $\mathcal{C}$ is said to be (Ricci Flow) invariant if $\mathcal{C}$ is preserved by Hamilton's $O D E \dot{\mathrm{R}}=2 Q(\mathrm{R})$. Namely, if $\mathrm{R}(t)$ is a solution to Hamilton's ODE on some time interval $[0, T)$ such that $\mathrm{R}(0) \in \mathcal{C}$, then $\mathrm{R}(t) \in \mathcal{C}$ for all $t \in[0, T)$.

Details and references about Hamilton's ODE are given in Section 2 ,

Hamilton's maximum principle for tensors ([17, Theorem 4.3]) implies that such a cone is preserved by Ricci flow in the sense that, if $\left(M, g_{0}\right)$ is a compact Riemannian manifold such that $\mathrm{R} \geq_{\mathcal{C}} 0$, then the Ricci flow $(M, g(t))$ such that $g(0)=g_{0}$ satisfies $\mathrm{R}(g(t)) \geq_{\mathcal{C}} 0$ as long as it exists.

We are now ready to state our result. It roughly says the following. We consider a manifold whose $\mathcal{C}$-curvature is bounded from below, where $\mathcal{C}$ is an invariant condition between nonnegative Ricci curvature and nonnegative curvature operator. We furthermore assume that an a priori estimate on the blow up rate of the scalar curvature of the Ricci flow as $t$ goes to zero is true. Then the $\mathcal{C}$-curvature can be bounded from below on a small time interval.

Theorem 1.3. For any dimension $n \in \mathbb{N}$, any $A \in\left(0, \frac{1}{4}\right)$ and any $B>0$, one can find $T=T(n, A, B)$ and $K=K(n, A, B)$ such that if $\mathcal{C} \subset S_{B}^{2} \Lambda^{2} \mathbb{R}^{n}$ is a closed convex cone which satisfies:

1. $\mathcal{C}$ is an invariant nonnegativity condition, 
2. $\mathcal{C}$ contains the cone of nonnegative curvature operators,

3. $\mathcal{C}$ is contained in the cone of curvature operators whose Ricci curvature is nonnegative,

and $\left(M^{n}, g(t)\right)_{t \in\left[0, T^{\prime}\right)}$ is a Ricci flow on a smooth compact manifold satisfying :

1. $\mathrm{R}(g(0)) \geq_{\mathcal{C}}-\varepsilon \mathrm{I}$ at $t=0$ for some $\varepsilon \in[0,1]$,

2. $|\operatorname{Scal}(g(t))| \leq A / t+B$ for $t$ in $\left(0, T^{\prime}\right)$,

we have :

$$
\mathrm{R}(g(t)) \geq_{\mathcal{C}}-K \varepsilon \mathrm{I}
$$

for all $t$ in $\left[0, T^{\prime}\right) \cap[0, T)$.

Remark 1.4. During the redaction of this article, the author has been informed that a similar estimate was also known by Miles Simon and Arthur Schlichting.

Example 1.5. Known examples of cones which satisfy the assumptions of the theorem include :

- the cone $\mathcal{C}_{C O}$ of nonnegative curvature operators,

- the cone $\mathcal{C}_{2 C O}$ of 2-nonnegative curvature operators,

- the cone $\mathcal{C}_{I C 1}$ of curvature operators which have nonnegative isotropic when extended by 0 to $\Lambda^{2} \mathbb{R}^{n+1}$,

- the cone $\mathcal{C}_{I C 2}$ of curvature operators which have nonnegative isotropic when extended by 0 to $\Lambda^{2} \mathbb{R}^{n+2}$.

All these conditions have been extensively studied $([4,[7,[8,[5])$ and compact manifolds with $\mathcal{C}$-nonnegative curvature have been classified when $\mathcal{C}$ is one of these four cones. An exposition of the relations between these condtions and how nonnegativity of these curvatures affect the topology of the underlying manifold can be found in the Brendle's book [6] together with precise definitions and additional references. It should also be noted that Wilking has given a unified proof of the preservation of these conditions (along with others) in 27.

Some continuous families of such cones have also been constructed in [4] and 14.

It should be noted that in dimension greater or equal to 4 , nonnegative Ricci curvature is not preserved, see 21 .

Remark 1.6. If $\mathcal{C}$ satisfies the assumptions of the theorem and moreover is a Wilking cone (see [27), it follows from the work of Gururaja, Maity and Seshadri in [16] that $\mathcal{C}$ is included in $\mathcal{C}_{I C 1}$.

From now on any curvature condition $\mathcal{C}$ is supposed to satisfy the assumptions of Theorem 1.3 .

The estimate of Theorem 1.3 allows us to adapt the methods of [26] and 25] to some higher dimensional situations. 
In the first two applications, the estimate on the scalar curvature which is required to apply Theorem 1.3 will be obtained by Perelman's pseudolocality theorem, first stated in [24] but omitting the crucial assumption of completeness as pointed by Topping, see [15, Theorem A.3], complete statement and proofs can be found in [20, 10].

Our first application is to show that, if the $\mathcal{C}$-curvature is bounded from below along a sequence of compact $n$-dimensional smooth manifolds which Gromov-Hausdorff converges (we will write GH-converges in the sequel) to a compact $n$-dimensional smooth manifold, then, up to a subsequence, the associated Ricci flows converge to a Ricci flow of the limit manifold (where the initial condition is to be understood in a weak sense). Here convergence is smooth convergence of the Ricci flows up to diffeomorphisms, as in 18. More precisely, we prove the following theorem, which is an higher dimensional analogue of [26. Theorem 9.2], where such a theorem has been proved in dimension 3 under lower bounds on the Ricci curvature and without assuming smoothness and compactness of the limit :

Theorem 1.7. Let $\left(M_{k}, g_{k}\right)$ be a sequence of compact $n$-manifolds which satisfies $R \geq_{\mathcal{C}}-\mathrm{I}$ and which $G H$-converges to a compact smooth $n$-manifold $(M, g)$. Let $\left(M_{k}, g_{k}(t)\right)_{t \in\left[0, T_{k}\right)}$ be the maximal solution of the Ricci flow satisfying $g_{k}(0)=$ $g_{k}$. Then :

1. there is a positive constant $T$ such that each Ricci flow $\left(M_{k}, g_{k}(t)\right)$ is defined at least on $[0, T)$ and the sequence of Ricci flows $\left(M_{k}, g_{k}(t)\right)_{t \in(0, T)}$ is precompact in the sense of Cheeger-Gromov-Hamilton.

2. any Cheeger-Gromov-Hamilton limit $(\tilde{M}, \tilde{g}(t))_{t \in(0, T)}$ of a convergent subsequence of $\left(M_{k}, g_{k}(t)\right)_{t \in(0, T)}$ is such that $\tilde{M}$ is homeomorphic to $M$ and the distance functions $d_{\tilde{g}(t)}$ uniformly converge as $t$ goes to 0 to some distance $\tilde{d}$ which is isometric to the distance $d_{g}$. In particular the $M_{k}$ 's are homeomorphic to $M$ for $k$ large enough.

Remark 1.8. Along the proof ot Theorem 1.7, we will see that the precompactness of the sequence of flows $\left(M_{k}, g_{k}(t)\right)_{t \in(0, T)}$ still holds when one replaces $\mathcal{C}$-curvature bounded from below by Ricci curvature bounded from below (see Lemma 4.1). However, our method of proof requires the lower bound on the $\mathcal{C}$-curvature to control the initial condition of the limit flow.

Remark 1.9. In the conclusions of the theorem, the fact that the $M_{k}$ 's are homeomorphic to $M$ for $k$ large enough can be seen using Cheeger and Colding's work on manifolds with Ricci curvature bounded from below (see [11, Theorem A.1.12]). Additionaly, Cheeger and Colding's result allow to strengthen the conclusion from homeomorphism to diffeomorphism. However, our proof is independent of this work.

Another application is a result about manifolds whith almost nonnegative $\mathcal{C}$-curvature, in the spirit of [26, Theorem 1.7] :

Theorem 1.10. For any $i>0$ and $D>0$, for any $n \in \mathbb{N}$, there is an $\varepsilon>0$ such that any manifold $(M, g)$ satisfying: 
1. $\operatorname{inj}(g) \geq i$

2. $\operatorname{diam}(M, g) \leq D$

3. $\mathrm{R} \geq_{\mathcal{C}}-\varepsilon \mathrm{I}$

admits a metric whose curvature is $\mathcal{C}$-nonnegative.

Using the classifcation results of Brendle [6, Theorem 9.33], Micallef and Wang [22, Theorem 3.1] and remark 1.6, if we moreover assume that $\mathcal{C}$ is a Wilking cone, we have that the universal cover of $M$ is diffeomorphic to a product $\mathbb{R}^{k} \times N_{1} \times \cdots \times N_{l}$ where each $N_{i}$ is one of the following :

- a standard sphere $\mathbb{S}^{n}$ with $n \geq 2$,

- a compact symmetric space.

We then impose stronger requirements on the cone $\mathcal{C}$. We assume that $\mathcal{C}$ is included in the cone of curvature operators whose sectional curvature is nonnegative. The cones which satisfy this assumptions in the list of Example 1.5 are $\mathcal{C}_{C O}$ and $\mathcal{C}_{I C 2}$.

This allows us to weaken the hypothesis of our results. In this context, it turns out that a convenient assumption that can be used to fulfill the hypothesis of Theorem 1.3 is that balls have almost euclidean volume. This is proved in Lemma [5.1, and was inspired to the author by the recent work of Cabezas-Rivas and Wilking [9. For instance, Theorem 1.7 becomes :

Theorem 1.11. Let $\mathcal{C}$ be a cone satifying the hypothesis of Theorem 1.3 and which is contained in the cone of curvature operator whose sectional curvature is nonnegative.

For any $n \in \mathbb{N}$, there exist $\kappa>0, T>0$ and $\delta>0$ such that if $(X, d)$ is a metric space which is a Gromov-Hausdorff limit of a sequence of compact manifolds $\left(M_{i}^{n}, g_{i}\right)$ such that:

- $\mathrm{R}\left(g_{i}\right) \geq_{\mathcal{C}}-\kappa \mathrm{I}$,

- for any $x \in M_{i}^{n}, \operatorname{vol}_{g_{i}}\left(B_{g_{i}}(x, 1)\right) \geq(1-\delta) \omega_{n}$, where $\omega_{n}$ is the volume of the unit ball in $\mathbb{R}^{n}$,

then one can find a Ricci flow $(M, g(t))$ defined on $(0, T)$ with bounded curvature on each time slice such that $M$ is homeomorphic to $X$ and the distance $d_{g}(t)$ converge uniformly on any compact of $M$ to a distance $\tilde{d}$ such that $(M, \tilde{d})$ is isometric to $(X, d)$.

Remark 1.12. The fact that $X$ is a manifold is a direct consequence of Perelman's stability theorem (see [19]), but we will not use this result in the proof. The metric space $(X, d)$ in our result is an Alexandrov space with curvature bounded form below and can have cone-like singularities, but the almost euclidean volume condition forbids too sharp cone angles.

Similarly, we get a stronger analogue of Theorem 1.10: 
Theorem 1.13. Let $\mathcal{C}$ be a cone satifying the hypothesis of Theorem 1.3 and which is contained in the cone of curvature operator whose sectional curvature is nonnegative.

For any $n \in \mathbb{N}$, there exists $\delta>0$ such that for any $D>0$, one can find $\varepsilon>0$ such that if $\left(M^{n}, g\right)$ is a compact Riemannian manifold such that:

- $\mathrm{R}(g) \geq_{\mathcal{C}}-\varepsilon \mathrm{I}$,

- $\forall x \in M, \operatorname{vol}_{g}\left(B_{g}(x, 1)\right) \geq(1-\delta) \omega_{n}$,

- $\operatorname{diam}(M, g) \leq D$,

then $M$ admits a metric with $\mathcal{C}$-nonnegative curvature.

The article is organised as follows : in Section 2 we set up the notations and give some background about the evolution equation of the curvature operator along the Ricci flow that will be used in the proof of Theorem 1.3. In Section 3. we give the proof of Theorem 1.3. The applications are discussed in Section 4. Section 5 is devoted to the applications in the case where $\mathcal{C}$-nonnegative curvature implies nonneagtive sectional curvature.

\section{Acknowledgements}

The author is grateful to Gilles Carron and Harish Seshadri for helpful discussions during the elaboration of this paper. The author also thanks his supervisor Gérard Besson for his interest and support.

\section{Preliminaries about algebraic curvature oper- ators.}

In this section, we set up the notations that will be used in this paper. Our conventions follow closely those of Böhm and Wilking in [4].

We will denote by $S^{2} \Lambda^{2} \mathbb{R}^{n}$ the vector space of symmetric operators on $\Lambda^{2} \mathbb{R}^{n}$ equiped with the standard inner product. $S_{B}^{2} \Lambda^{2} \mathbb{R}^{n}$ is the vector space of operators in $S^{2} \Lambda^{2} \mathbb{R}^{n}$ which in addition satisfy the first Bianchi identity. It is called the space of algebraic curvature operators on $\mathbb{R}^{n}$. As a norm on this space we use the classical Frobenius norm $\|\mathrm{R}\|^{2}=\operatorname{trace}\left(\mathrm{R}^{2}\right)$. Similar constructions hold on the tangent bundle of a Riemannian manifold $(M, g)$ and give rise to the bundles $S^{2} \Lambda^{2} T M$ and $S_{B}^{2} \Lambda^{2} T M$.

The curvature tensor of a manifold $(M, g)$ will always be viewed as a section of the bundle of curvture operators, $S_{B}^{2} \Lambda^{2} T M$. We follow the convention of [4] for the curvature operator, namely, the curvature operator of a round sphere of radius 1 is the identity.

We will use R, Ric and Scal to denote the curvature operator, Ricci curvature and scalar curvature. When considering a Ricci flow $(M, g(t))$, we will often not specify the dependence on $t$ of these various curvature when no confusion is 
possible. We will write I for the identity operator of $S_{B}^{2} \Lambda^{2} \mathbb{R}^{n}$ and id for the identity of $\mathbb{R}^{n}$.

Hamilton defined a bilinear map :

$$
\begin{aligned}
\#: S^{2} \Lambda^{2} \mathbb{R}^{n} \times S^{2} \Lambda^{2} \mathbb{R}^{n} & \rightarrow S^{2} \Lambda^{2} \mathbb{R}^{n} \\
(\mathrm{R}, \mathrm{L}) & \mapsto \mathrm{R} \# \mathrm{~L}
\end{aligned}
$$

whose expression can be found in [17] or 4].

If $g(t)$ is a family of metric on $M$ evolving along the Ricci flow, Hamilton showed in [17] that in appropriate coordinates the curvature operator $\mathrm{R}_{g(t)}$ satisfy the following evolution equation :

$$
\frac{\partial \mathrm{R}}{\partial t}=\Delta \mathrm{R}+2\left(\mathrm{R}^{2}+\mathrm{R}^{\#}\right),
$$

where $\Delta$ is the connection laplacian and $\mathrm{R}^{\#}=\mathrm{R} \# \mathrm{R}$.

Removing the laplacian in this evolution equation, we obtain Hamilton's ODE :

$$
\dot{\mathrm{R}}=2\left(\mathrm{R}^{2}+\mathrm{R}^{\#}\right)=2 Q(\mathrm{R}) .
$$

We will need the following algebraic fact about the \# operator, which was proved by Böhm and Wilking [4, Lemma 2.1] :

Proposition 2.1. $\mathrm{R}+\mathrm{R} \# \mathrm{I}=\mathrm{Ric} \wedge \mathrm{id}$

Here Ric $\wedge$ id is the curvature operator defined by, for any $u$ and $v$ in $\mathbb{R}^{n}$ :

$$
\operatorname{Ric} \wedge \operatorname{id}(u \wedge v)=\frac{1}{2}(\operatorname{Ric}(u) \wedge v+u \wedge \operatorname{Ric}(v)),
$$

where Ric is viewed as an operator on $\mathbb{R}^{n}$. In particular, if $\left(\lambda_{i}\right)_{1 \leq i \leq n}$ are the eigenvalues of Ric then the eigenvalues of $\operatorname{Ric} \wedge$ id are $\left(\frac{\lambda_{i}+\lambda_{j}}{2}\right)_{1 \leq i<j \leq n}$.

\section{Proof of Theorem 1.3.}

Proof of Theorem 1.3. According to our hypothesis, if we define a new section of the bundle of curvature operators L by :

$$
\mathrm{L}=\mathrm{R}+\varepsilon(\varphi(t)+t \alpha \mathrm{Scal}) \mathrm{I},
$$

it is enough to find a positive smooth function $\varphi$, a constant $\alpha$ and a time $T>0$, all depending only on $A$ and $B$ such that $\mathrm{L} \in \mathcal{C}$ for $t \in[0, T)$. The fact that $t$ Scal and $\varphi$ are unformly bounded on $[0, T]$ will then give the required bound. To ensure that $\mathrm{L} \in \mathcal{C}$ at time 0 , we impose that $\varphi(0)=1$. Since such lower bounds are likely to get worse with time, we will assume that $\varphi^{\prime} \geq 0$.

To prove that $\mathrm{L}$ remains in $\mathcal{C}$, we will apply Hamilton's maximum principle for tensors [17, or more precisely a variant of it called maximum principle with avoidance set proved by $\mathrm{Chow}$ and $\mathrm{Lu}$ in [12, Theorem 4]. This variant allows 
us to use our a priori estimate on the scalar curvature (which is not implied by the ODE) in the study of the ODE associated to the PDE satisfied by L.

We will impose conditions on $\varphi$ and $\alpha$ during the proof and verify that these conditions can be fullfilled at the end of the proof.

We first compute the evolution of L :

$$
\begin{aligned}
\frac{\partial \mathrm{L}}{\partial t} & =\Delta \mathrm{R}+2 Q(\mathrm{R})+\varepsilon\left(\varphi^{\prime}+\alpha \mathrm{Scal}+t \alpha\left(\Delta \mathrm{Scal}+2|\mathrm{Ric}|^{2}\right)\right) \mathrm{I} \\
& =\Delta \mathrm{L}+2 Q(\mathrm{R})+\varepsilon\left(\varphi^{\prime}+\alpha \mathrm{Scal}+2 t \alpha|\mathrm{Ric}|^{2}\right) \mathrm{I} \\
& =\Delta \mathrm{L}+2 N(\mathrm{~L}) .
\end{aligned}
$$

We now have to show that $\mathcal{C}$ is preserved by the differential equation $\dot{\mathrm{L}}=$ $2 N(\mathrm{~L})$. That is, given $\mathrm{L} \in \partial \mathcal{C}$, we need to show that $N(\mathrm{~L}) \in \mathcal{C}$. Since $\mathcal{C}$ is preserved by Hamilton's ODE, we know that $Q(\mathrm{~L}) \in \mathcal{C}$ and we just need to show (since $\mathcal{C}$ is convex) that $D(\mathrm{~L})=N(\mathrm{~L})-Q(\mathrm{~L}) \in \mathcal{C}$. This idea comes from the work of Böhm and Wilking in [4].

We will in fact prove that $D(\mathrm{~L})$ is a nonnegative curvature operator, which will be enough since $\mathcal{C}$ contains the cone of nonnegative curvature operator.

Using Böhm and Wilking identity (proposition 2.1), we have :

$$
\begin{aligned}
Q(\mathrm{~L}) & =Q(\mathrm{R})+2 \varepsilon(\varphi+t \alpha \mathrm{Scal})(\mathrm{R}+\mathrm{R} \# \mathrm{I})+\varepsilon^{2}(\varphi+t \alpha \mathrm{Scal})^{2} Q(\mathrm{I}) \\
& =Q(\mathrm{R})+2 \varepsilon(\varphi+t \alpha \mathrm{Scal})(\mathrm{Ric} \wedge \mathrm{id})+(n-1) \varepsilon^{2}(\varphi+t \alpha \mathrm{Scal})^{2} \mathrm{I} .
\end{aligned}
$$

We then compute $D(\mathrm{~L})$ :

$$
\begin{aligned}
D(\mathrm{~L})= & N(\mathrm{~L})-Q(\mathrm{~L}) \\
= & \frac{\varepsilon}{2}\left(\varphi^{\prime}+\alpha \text { Scal }+2 t \alpha \mid \text { Ric }\left.\right|^{2}\right) \mathrm{I} \\
& -2 \varepsilon(\varphi+t \alpha \text { Scal })(\operatorname{Ric} \wedge \mathrm{id})-(n-1) \varepsilon^{2}(\varphi+t \alpha \text { Scal })^{2} \mathrm{I} .
\end{aligned}
$$

In order to estimate the $2 \operatorname{Ric} \wedge$ id term, we use that $L \in \mathcal{C}$ has nonnegative Ricci curvature, which gives that Ric $\geq-(n-1) \varepsilon(\varphi+t \alpha$ Scal $)$ id as symmetric operators. Since trace $(\mathrm{Ric})=$ Scal, we have :

$$
-(n-1) \varepsilon(\varphi+t \alpha \text { Scal }) \text { id } \leq \text { Ric } \leq\left(\text { Scal }+(n-1)^{2} \varepsilon(\varphi+t \alpha \text { Scal })\right) \text { id } .
$$

This implies :

$$
2 \operatorname{Ric} \wedge \mathrm{id} \leq\left(\mathrm{Scal}+(n-1)^{2} \varepsilon(\varphi+t \alpha \text { Scal })\right) \mathrm{I} .
$$

We now assume that:

$$
\varphi+t \alpha \text { Scal } \geq 0 \quad \text { condition }(\mathrm{C} 1) .
$$

This allows us to estimate $D(\mathrm{~L})$ :

$$
\begin{aligned}
D(\mathrm{~L}) \geq & \frac{\varepsilon}{2}\left(\varphi^{\prime}+\alpha \text { Scal }\right) \mathrm{I} \\
& -\varepsilon(\varphi+t \alpha \mathrm{Scal})\left(\mathrm{Scal}+(n-1)^{2} \varepsilon(\varphi+t \alpha \text { Scal })\right) \mathrm{I} \\
& -(n-1) \varepsilon^{2}(\varphi+t \alpha \mathrm{Scal})^{2} \mathrm{I} .
\end{aligned}
$$


We rearrange the terms in the following way 1 :

$$
\begin{aligned}
D(\mathrm{~L}) \geq & \frac{\varepsilon}{2} \varphi^{\prime} \\
& +\varepsilon \operatorname{Scal}\left(\left(\frac{1}{2}-t \mathrm{Scal}\right) \alpha-\varphi\right) \\
& -(2 n-1)(n-1) \varepsilon^{2}(\varphi+t \alpha \mathrm{Scal})^{2}
\end{aligned}
$$

We now assume that :

$$
0 \leq\left(\frac{1}{2}-t \text { Scal }\right) \alpha-\varphi \leq 1 \quad \text { condition }(\mathrm{C} 2),
$$

Since Scal $\geq-\varepsilon n(n-1)$ at $t=0$, it remains so as long as the solution exists. Therefore we have :

$$
\begin{aligned}
D(\mathrm{~L}) \geq & \frac{\varepsilon}{2} \varphi^{\prime} \\
& -\varepsilon^{2} n(n-1) \\
& -(2 n-1)(n-1) \varepsilon^{2}(\varphi+t \alpha \mathrm{Scal})^{2},
\end{aligned}
$$

and since $\varepsilon \in[0,1]$ :

$$
\begin{aligned}
\frac{1}{\varepsilon^{2}} D(\mathrm{~L}) \geq & \frac{\varphi^{\prime}}{2} \\
& -n(n-1) \\
& -(2 n-1)(n-1)(\varphi+t \alpha \mathrm{Scal})^{2} .
\end{aligned}
$$

We now use that $\mid t$ Scal $\mid \leq A+B t$ to get :

$$
\begin{aligned}
\frac{1}{\varepsilon^{2}} D(\mathrm{~L}) \geq & \frac{\varphi^{\prime}}{2} \\
& -n(n-1) \\
& -(n-1)(2 n-1)(\varphi+\alpha(A+B t))^{2} .
\end{aligned}
$$

To ensure that $D(\mathrm{~L})$ is a nonnegative operator, it is then enough to show that :

$$
\frac{\varphi^{\prime}}{2}-n(n-1)-(n-1)(2 n-1)(\varphi+\alpha(A+B t))^{2} \geq 0 \quad \text { condition }(\mathrm{C} 3) .
$$

We now have to find $\varphi, \alpha$ and $T$ such that conditions (C1), (C2) and (C3) are satisfied on $[0, T]$.

Using again that $-n(n-1) t \leq t$ Scal $\leq A+B t$, we have that conditions $(\mathrm{C} 1)$ and (C2) are implied by the following inequalities which involves only $A$, $B$ and the dimension $n$ :

$$
\left.\begin{array}{l}
\left(\frac{1}{2}-(A+B t)\right) \alpha-\varphi \geq 0 \\
\left(\frac{1}{2}+t n(n-1)\right) \alpha-\varphi \leq 1 \\
\varphi-n(n-1) t \alpha \geq 0
\end{array}\right\}
$$

\footnotetext{
${ }^{1}$ We will drop the I's in the next inequalities, here a real number $\alpha$ should be viewed as the operator $\alpha \mathrm{I}$.
} 
Looking at conditions (C4) at $t=0$, we see that it is fulfilled if $\alpha$ belongs to $\left[\frac{2}{1-2 A}, 4\right]$. We now impose that $A<\frac{1}{4}$. Let $\alpha \in\left(\frac{2}{1-2 A}, 4\right)$, and $\varphi(t)=1+\beta t$. Conditition (C4) is then satisfied at time 0 with strict inequalities.

We now choose $\beta$ big enough such that condition (C3) is fulfilled with a strict inequality. By continuity of $\varphi$, these conditions are still fulfilled for $t$ in some small time interval $[0, T)$.

Our choices of $\varphi, \alpha$ and $T$ depend only on $A, B$ and $n$, the theorem is then proved.

\section{First applications.}

\subsection{Gromov-Hausdorff converging sequences whose $\mathcal{C}$-curvature is bounded from below.}

In this section, we prove Theorem 1.7. We first state a lemma which is of independent interest, the idea of using pseudolocality and convergence of the isoperimetric profiles in the proof of the following lemma was suggested to the author by Gilles Carron :

Lemma 4.1. Let $\left(M_{k}, g_{k}\right)_{k \in \mathbb{N}}$ be a sequence of smooth compact $n$-dimensional Riemannian manifold which satisfies $\operatorname{Ric}\left(g_{k}\right) \geq-(n-1) g_{k}$ and which GHconverges to a smooth compact $n$-dimensional Riemannian manifold $(M, g)$.

Then for every every $A>0$, there exist $k_{0} \in \mathbb{N}, B>0$ and $T>0$ such that, for any $k \geq k_{0}$ the Ricci flows $\left(M_{k}, g_{k}(t)\right)$ whith initial condition $\left(M_{k}, g_{k}\right)$ exist at least on $[0, T)$ and satisfy :

1. $\left\|\mathrm{R}\left(g_{k}(t)\right)\right\| \leq A / t+B$ for all $t \in(0, T)$,

2. $\operatorname{vol}\left(B_{g_{k}(t)}(x, \sqrt{t})\right) \geq c t^{n / 2}$ for all $t \in(0, T)$ and $x \in M_{i}$.

In particular, the Ricci flows $\left(M_{k}, g_{k}(t)\right)_{t \in(0, T)}$ form a precompact sequence in the sense of Cheeger Gromov and Hamilton.

Proof. We want to apply Perelman's pseudolocality (24, Section 10], 20, Theorem 30.1, Corollary 35.1]) to get the two estimates of the lemma. The precompactness statement then follows from Hamilton's compactness theorem 18.

Let $A>0$ be fixed. We already know that for any $x \in M_{k}, \operatorname{Scal}_{g_{k}}(x) \geq$ $-n(n-1)$. Thus we just need to find some $r_{0} \in\left(0,(n(n-1))^{-1 / 2}\right]$ such that any smooth domain $\Omega$ contained in a ball of radius $r_{0}$ in $M_{k}$ for $k$ large enough satisfies the almost Euclidean isoperimetric estimate :

$$
|\partial \Omega|^{\frac{n}{n-1}} \geq(1-\delta) \gamma_{n}|\Omega|
$$

where $\gamma_{n}$ is the euclidean isoperimetric constant and $\delta$ is given by the pseudolocality theorem.

To obtain this estimate, we will consider the isoperimetric profiles of the $\left(M_{k}, g_{k}\right)$ 's, that will be denoted by $h_{k}(\beta)$. Since $(M, g)$ is smooth, by a result of Bérard and Meyer [3, Appendice C], its isoperimetric profile $h(\beta)$ is equivalent 
to the euclidean one as $\beta$ goes to zero. Thus we can find, for any given $\varepsilon>0$, some $\rho>0$ such that :

$$
\beta<\rho \Rightarrow h(\beta) \geq(1-\varepsilon) \frac{\gamma_{n}}{\operatorname{vol}(M, g)^{\frac{1}{n}}} \beta^{\frac{n-1}{n}}
$$

We then use a result from Bayle thesis [2] : under non collapsing GHconvergence to a smooth manifold with Ricci curvature bounded from below, the ratio of the isoperimetric profiles $h_{k} / h$ is going to 1 uniformly on $(0,1)$. Then, for $i$ large enough :

$$
h_{k} \geq(1-\varepsilon) h .
$$

Let $\Omega \subset M_{i}$ be a smooth domain whose volume is less than $\rho \operatorname{vol}\left(M_{k}, g_{k}\right)$. We then have :

$$
\begin{aligned}
|\partial \Omega| & \geq \operatorname{vol}(M, g) \times h_{k}\left(\frac{|\Omega|}{\operatorname{vol}(M, g)}\right) \\
& \geq \operatorname{vol}(M, g) \times(1-\varepsilon) h\left(\frac{|\Omega|}{\operatorname{vol}(M, g)}\right) \\
& \geq \operatorname{vol}(M, g) \times(1-\varepsilon)^{2} \frac{\gamma_{n}}{\operatorname{vol}(M, g)^{\frac{1}{n}}}\left(\frac{|\Omega|}{\operatorname{vol}(M, g)}\right)^{\frac{n-1}{n}} \\
& =(1-\varepsilon)^{2} \gamma_{n}|\Omega|^{\frac{n-1}{n}}
\end{aligned}
$$

If we take $\varepsilon$ small enough, we get estimate (11) for domains of volume less then $\rho \operatorname{vol}\left(M_{k}, g_{k}\right)$.

Now, using Colding's theorem on the continuity of volume [13, for $k$ large enough, $\operatorname{vol}\left(M_{k}, g_{k}\right) \geq V / 2$ where $V$ is the volume of $(M, g)$. In particular, our almost Euclidean isoperimetric inequality is valid for domains of volume less than $\rho V / 2$. Since the Ricci curvature is bounded from below, Bishop Gromov inequality gives us that:

$$
\operatorname{vol}\left(B_{g_{k}}(x, r)\right) \leq V_{-1}(r)
$$

where $V_{-1}(r)$ is the volume a radius $r$ ball in the $n$-dimensional hyperbolic space. This shows that our isoperimetric inequality is valid for domains included in balls of radius less than $r_{0}$ where $r_{0}$ is such that $V_{-1}\left(r_{0}\right)=\rho V / 2$.

Finally, pseudolocality applies and we get the required bounds.

We now proove Theorem 1.7

Proof of Theorem 1.7. We now consider a sequence $\left(M_{k}^{n}, g_{k}\right)$ of smooth compact manifolds whose $\mathcal{C}$-curvature is bounded from below by $-\mathrm{I}$ and which in addition satisfy the assumptions of Lemma 4.1 .

Thanks to the previous lemma, we can find $i_{0} \in \mathbb{N}, T>0$ and a constant $B$ such that, for $k \geq k_{0}$, the Ricci flows $\left(M_{k}, g_{k}(t)\right)$ satisfying $g_{k}(0)=g_{k}$ satisfy :

$$
\left|\operatorname{Scal}\left(g_{k}(t)\right)\right| \leq \frac{1}{8 t}+B \text { for } t \in(0, T) .
$$


We now use Theorem 1.3 and the fact that $\left(M_{k}, g_{k}(0)\right)$ has $\mathcal{C}$-curvature bounded from below by $-\mathrm{I}$ to find $T^{\prime}>0$ and $K>0$ such that, for $t \in\left(0, T^{\prime}\right)$,

$$
\mathrm{R}\left(g_{k}(t)\right) \geq_{\mathcal{C}}-K \mathrm{I} .
$$

Since this implies that the Ricci curvature of $\left.\left(M_{k}, g_{k}(t)\right)\right)$ is bounded from below by $-(n-1) K$ on $\left[0, T^{\prime}\right)$, we can apply Lemma 6.1 in 25 . We get, for some constant $c>0$, that for $k \geq k_{0}, x, y \in M_{k}$ and $0<s \leq t<T^{\prime}$ :

$$
d_{g_{k}(s)}(x, y)-c(\sqrt{t}-\sqrt{s}) \leq d_{g_{k}(t)} \leq e^{c(t-s)} d_{g_{k}(s)}
$$

where $d_{g_{k}(t)}$ is the distance function of $\left(M_{k}, g_{k}(t)\right)$.

Consider now a subsequence of the sequence $\left(M_{k}, g_{k}(t)\right)_{t \in\left(0, T^{\prime}\right)}$ which converges in the sense of Cheeger-Gromov-Hamilton to a Ricci flow $(\tilde{M}, \tilde{g}(t))_{t \in\left(0, T^{\prime}\right)}$. This flow also satisfies estimates (2), (3) and (4).

As in the proof of Theorem 9.2 in [25], we can prove that the distances $d_{\tilde{g}(t)}$ uniformly converge as $t$ goes to zero to some distance $\tilde{d}$, which define the usual manifold topology on $\tilde{M}$, and that $(\tilde{M}, \tilde{d})$ is isometric to the GH-limit $(M, g)$ of the sequence $\left(M_{k}, g_{k}\right)$. In particular, $M$ and $\tilde{M}$ are homeomorphic.

\subsection{Manifolds with almost nonnegative $\mathcal{C}$-curvature.}

We now proove Theorem 1.10 .

Proof. By contradiction, take a sequence of counterexamples $\left(M_{k}, g_{k}\right)$ satisfying $\mathrm{R} \geq_{\mathcal{C}}-\varepsilon_{k} \mathrm{I}$, where $\varepsilon_{k}$ goes to 0 , and the required bounds on the diameter and injectivity radius. We assume that none of the $M_{k}$ admits a metric with nonnegative $\mathcal{C}$-curvature. Without loss of generality, we assume that $\varepsilon_{k} \leq 1$.

Since the injectivity radius and the Ricci curvature are bounded from below, we can use Anderson-Cheeger theorem [1, Theorem 0.3]. It gives us, for any $\varepsilon>0$, some $r>0$ such that every ball $B$ of radius less than $r_{0}$ admit an harmonic coordinate chart $\varphi_{B}: B \rightarrow \mathbb{R}^{n}$ with :

$$
\frac{1}{1+\varepsilon} \varphi_{B}^{*} \delta \leq g \leq(1+\varepsilon) \varphi_{B}^{*} \delta
$$

on $B$, where $\delta$ is the euclidean metric on $\mathbb{R}^{n}$.

If we choose $\varepsilon$ small enough, this control will give us an almost Euclidean isoperimetric estimate on balls of radius less than $r_{0}$.

Consider the sequence of Ricci flows $\left(M_{k}, g_{k}(t)\right)$ such that $g_{k}(0)=g_{k}$. Pseudolocality gives :

- each $\left(M_{k}, g_{k}(t)\right)$ exists at least on $[0, T)$ where $T$ does not depend on $k$,

- for $t \in(0, T),\left|\operatorname{Scal}\left(g_{k}(t)\right)\right| \leq \frac{1}{8 t}+B$, where $B$ does not depend on $k$.

- the Ricci flows $\left(M_{k}, g_{k}(t)\right)_{t \in(0, T)}$ form a precompact sequence in the sense of Cheeger-Gromov-Hamilton. 
We can then apply Theorem 1.3 to have that on some time interval $\left(0, T^{\prime}\right) \subset$ $(0, T), \mathrm{R}\left(g_{k}(t)\right) \geq_{\mathcal{C}}-K \varepsilon_{k} \mathrm{I}$.

Let $(M, g(t))_{t \in\left(0, T^{\prime}\right)}$ be the limit of a convergent subsequence of $\left(M_{k}, g_{k}(t)\right)_{t \in\left(0, T^{\prime}\right)}$, it satisfies $\mathrm{R}(g(t)) \geq_{\mathcal{C}} 0$ for $t \in\left(0, T^{\prime}\right)$. Now, since the Ricci curvature is bounded from below in time and along the sequence, we can find some constant $C$ such that :

$$
\operatorname{diam}\left(M_{k}, g_{k}(t)\right) \leq e^{C t} \operatorname{diam}\left(M_{k}, g_{k}(t)\right) \leq e^{C t} D
$$

for all $k \in \mathbb{N}$ and $t \in\left(0, T^{\prime}\right)$.

This implies that $M$ is compact. Hence, we have a subsequence of $\left(M_{k}, g_{k}\right)$ all of whose elements are diffeomorphic to $M$, in particular, these elements admit a metric with non-negative $\mathcal{C}$-curvature. This is a contradiction.

\section{Stronger results when operators in $\mathcal{C}$ have non- negative sectional curvature}

We now assume that $\mathcal{C}$ contains the cone of curvature operators whose sectional curvature is nonnegative.

As in the previous proofs, the crucial point is to get an $A / t+B$ bound on the scalar curvature. We first state a lemma which gives this bound when one has almost euclidean volume and $\mathcal{C}$-curvature bounded from below at the initial time. This lemma is a stronger version of Proposition 5.5 in [9].

Lemma 5.1. For any dimension $n$, any $A \in\left(0, A_{0}(n)\right)$, there exists $\kappa>0$, $\delta>0, \tilde{\kappa}>0$ and $T>0$ such that if $\left(M^{n}, g\right)$ is a compact Riemannian manifold such that:

- $\mathrm{R} \geq_{\mathcal{C}}-\kappa \mathrm{I}$,

- $\forall x \in M^{n}, \quad \operatorname{vol}_{g}\left(B_{g}(x, 1)\right) \geq(1-\delta) \omega_{n}$,

where $\omega_{n}$ is the volume of the unit ball in $\mathbb{R}^{n}$.

Then the Ricci flow $\left(M^{n}, g(t)\right)$ with initial condition $\left(M^{n}, g\right)$ exists at least on $[0, T)$ and satisfies:

- $\forall t \in(0, T) \quad\|\mathrm{R}(g(t))\| \leq \frac{A}{t}$,

- $\forall t \in(0, T) \quad \mathrm{R}(g(t)) \geq-\tilde{\kappa} \mathrm{I}$.

Proof. Since $\mid$ Scal $\mid \leq 2 \sqrt{n}\|\mathrm{R}\|$, we set $A_{0}(n)=\frac{1}{8 \sqrt{n}}$. This ensures that if $\|\mathrm{R}\| \leq \frac{A}{t}$ we have the right estimate on the scalar curvature to apply Theorem 1.3

The proof goes by contradiction. Fix $n \in \mathbb{N}$ and $A<A_{0}(n)$. Assume we can find a sequence of manifolds $\left(M_{i}, g_{i}\right)_{i \in \mathbb{N}}$ such that :

- $\mathrm{R}\left(g_{i}\right) \geq_{\mathcal{C}}-\delta_{i} \mathrm{I}$,

- $\forall x \in M, \quad \operatorname{vol}_{g_{i}}\left(B_{g_{i}}(x, 1)\right) \geq\left(1-\delta_{i}\right) \omega_{n}$, 
for some sequence $\left(\delta_{i}\right)_{i \in \mathbb{N}}$ going to zero. And assume furthermore that the sequence $\left(t_{i}\right)_{i \in \mathbb{N}}$ defined by $t_{i}=\sup \left\{t>0 \mid \forall s \leq t, s\left\|R\left(g_{i}(s)\right)\right\| \leq A\right\}$ goes to zero. Taking $i$ large enough, we can assume that $\delta_{i} \leq 1$ and $t_{i}$ is less than the time $T$ given by Theorem 1.3 , this ensures that for all $t \in\left[0, t_{i}\right]$ :

$$
\mathrm{R}\left(g_{i}(t)\right) \geq_{\mathcal{C}}-K \delta_{i} \mathrm{I} .
$$

With this lower bound, we can repeat word for word the proof of Proposition 5.5 in [9] and get the $\frac{A}{t}$ bound on the norm of the curvature operator on some time interval. The lower bound on $\mathcal{C}$-curvature is now given by Theorem 1.3 .

We now proove Theorem 1.11. The Ricci flow of $(X, d)$ is constructed as limit of the Ricci flows of the $\left(M_{i}, g_{i}\right)$, as in 25 .

Proof (of Theorem 1.11). Fix $\kappa$ and $\delta$ such that Lemma 5.1 apply with $A=$ $\frac{1}{16 \sqrt{n}}$. Consider a sequence $\left(M_{i}, g_{i}\right)$ satifying the assumption of the theorem. Using Lemma 5.1, we have that the Ricci flows $\left(M_{i}, g_{i}(t)\right)$ exist at least on $[0, T)$ and satisfy, for any $t$ in $[0, T)$ :

- $\left\|\mathrm{R}\left(g_{i}(t)\right)\right\| \leq \frac{1}{16 \sqrt{n} t}$,

- $\mathrm{R}\left(g_{i}(t)\right) \geq_{\mathcal{C}}-K \mathrm{I}$.

In addition, at time $t=0$, we have that any unit ball in any of the $M_{i}$ 's has volume at least $(1-\delta) \omega_{n}$. This allow us to apply Lemma 6.1 and Corolarry 6.2 in [25] to get that, on some possibly smaller time interval $\left[0, T^{\prime}\right]$, we have the estimates, for some constant $C>0$ depending only on $\kappa$ and $\delta$ :

- $\forall x \in M_{i}, \operatorname{vol}_{g_{i}}\left(B_{g_{i}}(x, 1)\right) \geq \frac{(1-\delta) \omega_{n}}{2}$,

- for $0<s \leq t \leq T^{\prime}, d_{g_{i}(s)}-C(\sqrt{t}-\sqrt{s}) \leq d_{g_{i}(t)} \leq e^{C(t-s)} d_{g_{i}(s)}$,

where $d_{g_{i}(t)}$ is the distance on $M_{i}$ induced by the metric $g_{i}(t)$.

We then argue as in the proof of Theorem 9.2 in [25] and get that the sequence of Ricci flows $\left(M_{i}, g_{i}(t)\right)_{t \in\left(0, T^{\prime}\right)}$ has a convergent subsequence whose limit $(M, g(t))_{t \in\left(0, T^{\prime}\right)}$ is a Ricci flow of the Gromov-Hausdorff limit $(X, d)$ of the sequence $\left(M_{i}, g_{i}\right)$ in the sense that it satisfies the conclusions of Theorem 1.11 .

We now go on with Theorem 1.13 ,

Proof of Theorem 1.13, Let $\delta>0$ and $\kappa>0$ be the constants given by Lemma 5.1] with $A=\frac{1}{16 \sqrt{n}}$. Fix $D>0$.

As in the proof of Theorem 1.10, consider a sequence of manifolds $\left(M_{i}, g_{i}\right)$ with :

- $\forall x \in M_{i}, \operatorname{vol}_{g_{i}}\left(B_{g_{i}}(x, 1)\right) \geq(1-\delta) \omega_{n}$,

- $\mathrm{R} \geq_{\mathcal{C}}-\varepsilon_{i} \mathrm{I}$, 
- $\operatorname{diam}\left(M_{i}, g_{i}\right) \leq D$,

where $\varepsilon_{i}$ goes to 0 as $i$ goes to infinity. Assume furthermore that none of the $M_{i}$ admits a metric with nonnegative $\mathcal{C}$-curvature. Without loss of generality, we can assume that $\varepsilon_{i} \leq \min (\kappa, 1)$.

Arguing as in the proofs of Theorem 1.11 and Theorem 1.10, we get that the Ricci flows $\left(M_{i}, g_{i}(t)\right)$ starting at $\left(M_{i}, g_{i}\right)$ exist at least on $[0, T)$, form a precompact family in the sense of Cheeger-Gromov-Hamilton, and satisfy :

- $\mathrm{R} \geq_{\mathcal{C}}-K \varepsilon_{i} \mathrm{I}$,

- $\operatorname{diam}\left(M_{i}, g_{i}(t)\right) \leq e^{C t} D$.

In particular any limit of a convergent subsequence will be compact and have nonnegative $\mathcal{C}$-curvature. Thus the sequence contains manifolds which admit metrics with $\mathcal{C}$-nonnegative curvature. This is a contradiction.

\section{References}

[1] Michael T. Anderson and Jeff Cheeger. $C^{\alpha}$-compactness for manifolds with Ricci curvature and injectivity radius bounded below. J. Differential Geom., 35(2):265-281, 1992.

[2] Vincent Bayle. Propriétés de concavité du profil isopérimétrique et applications. $\mathrm{PhD}$ thesis, Université Joseph Fourier, 2003. http://tel.archives-ouvertes.fr/tel-00004317/en/.

[3] Pierre Bérard and Daniel Meyer. Inégalités isopérimétriques et applications. Ann. Sci. École Norm. Sup. (4), 15(3):513-541, 1982.

[4] Christoph Böhm and Burkhard Wilking. Manifolds with positive curvature operators are space forms. Ann. of Math. (2), 167(3):1079-1097, 2008.

[5] Simon Brendle. A general convergence result for the Ricci flow in higher dimensions. Duke Math. J., 145(3):585-601, 2008.

[6] Simon Brendle. Ricci flow and the sphere theorem, volume 111 of Graduate Studies in Mathematics. American Mathematical Society, Providence, RI, 2010.

[7] Simon Brendle and Richard Schoen. Manifolds with 1/4-pinched curvature are space forms. J. Amer. Math. Soc., 22(1):287-307, 2009.

[8] Simon Brendle and Richard M. Schoen. Classification of manifolds with weakly 1/4-pinched curvatures. Acta Math., 200(1):1-13, 2008.

[9] E. Cabezas-Rivas and B. Wilking. How to produce a Ricci Flow via Cheeger-Gromoll exhaustion. ArXiv e-prints, July 2011. 
[10] Albert Chau, Luen-Fai Tam, and Chengjie Yu. Pseudolocality for the Ricci flow and applications. Canad. J. Math., 63(1):55-85, 2011.

[11] Jeff Cheeger and Tobias H. Colding. On the structure of spaces with Ricci curvature bounded below. I. J. Differential Geom., 46(3):406-480, 1997.

[12] Bennett Chow and Peng Lu. The maximum principle for systems of parabolic equations subject to an avoidance set. Pacific J. Math., 214(2):201-222, 2004.

[13] Tobias H. Colding. Ricci curvature and volume convergence. Ann. of Math. (2), 145(3):477-501, 1997.

[14] X. Gao and Y. Zheng. An Interpolating Curvature Condition Preserved By Ricci Flow. ArXiv e-prints, May 2011.

[15] G. Giesen and P. M. Topping. Ricci flows with unbounded curvature. ArXiv e-prints, June 2011.

[16] H. A. Gururaja, S. Maity, and H. Seshadri. On Wilking's criterion for the Ricci flow. ArXiv e-prints, January 2011.

[17] Richard S. Hamilton. Four-manifolds with positive curvature operator. $J$. Differential Geom., 24(2):153-179, 1986.

[18] Richard S. Hamilton. A compactness property for solutions of the Ricci flow. Amer. J. Math., 117(3):545-572, 1995.

[19] Vitali Kapovitch. Perelman's stability theorem. In Surveys in differential geometry. Vol. XI, volume 11 of Surv. Differ. Geom., pages 103-136. Int. Press, Somerville, MA, 2007.

[20] Bruce Kleiner and John Lott. Notes on Perelman's papers. Geom. Topol., 12(5):2587-2855, 2008.

[21] Davi Máximo. Non-negative Ricci curvature on closed manifolds under Ricci flow. Proc. Amer. Math. Soc., 139(2):675-685, 2011.

[22] Mario J. Micallef and McKenzie Y. Wang. Metrics with nonnegative isotropic curvature. Duke Math. J., 72(3):649-672, 1993.

[23] Huy T. Nguyen. Isotropic curvature and the Ricci flow. Int. Math. Res. Not. IMRN, (3):536-558, 2010.

[24] G. Perelman. The entropy formula for the Ricci flow and its geometric applications. ArXiv Mathematics e-prints, November 2002.

[25] M. Simon. Ricci flow of non-collapsed 3-manifolds whose Ricci curvature is bounded from below. ArXiv e-prints, March 2009.

[26] Miles Simon. Ricci flow of almost non-negatively curved three manifolds. J. Reine Angew. Math., 630:177-217, 2009. 
[27] B. Wilking. A Lie algebraic approach to Ricci flow invariant curvature conditions and Harnack inequalities. ArXiv e-prints, November 2010.

Institut Fourier, 100 RUE DES MATHS, 38402 St Martin D'HÈres.

Email adress: thomas.richard@ujf-grenoble.fr 